\title{
Instagram como herramienta de salud pública: un estudio sobre América del Sur
}

\author{
Instagram as a tool for public health: a study of South America ${ }^{1}$
}

\author{
Dra. Pâmela Araujo Pinto \\ Universidad de Aveiro | Campus Universitário de Santiago, 3810-193 Aveiro | Portugal \\ | https://orcid.org/0000-0002-9699-7073 | pinpamela@gmail.com
}

\section{Dra. Maria João Antunes}

Universidad de Aveiro | Campus Universitário de Santiago, 3810-193 Aveiro | Portugal

| https://orcid.org/0000-0002-7819-4103| mariajoao@ua.pt

\author{
Dra. Ana Margarida Pisco Almeida \\ Universidad de Aveiro | Campus Universitário de Santiago, 3810-193 Aveiro | Portugal \\ | https://orcid.org/0000-0002-7349-457X| marga@ua.pt
}

Fechas | Recepción: 15/04/2021 | Aceptación: 07/06/2021

\section{Resumen}

Entre 2015 y 2020, la adhesión de las autoridades sanitarias sudamericanas a Instagram ha aumentado. En 2015, de un total de 12 países, había tres perfiles activos, y en 2019, nueve. Sin embargo, son pocos los estudios que analizan el uso de Instagram como herramienta estratégica en el contexto de la salud pública en los países en desarrollo o de bajos ingresos. Así pues, este estudio pretende trazar un mapa de los perfiles de Instagram de las autoridades sanitarias de América del Sur. Para ello se realizó un estudio exploratorio basado en una revisión de la literatura y un análisis de contenido de estos perfiles. Se efectuó un análisis cualitativa y cuantitativa sobre los usos de la plataforma

\section{Abstract}

Between 2015 and 2020, the adherence of South American health authorities to Instagram has increased. In 2015, from a total of 12 countries, there were three active profiles and, in 2019, there were nine. However, a few studies analyze the use of Instagram as a strategic tool in the context of public health in developing or low-income countries. Therefore, this study aims to map health authorities' Instagram profiles in South America. An exploratory study was carried out based on a literature review and a content analysis of these profiles. It conducts a qualitative and quantitative analysis on the uses of the platform to expand information related to public health. The sample is composed of 2,418 posts,

\footnotetext{
${ }^{1}$ Traducción realizada por Lersi Duran
} 
para ampliar la información relacionada con la salud pública. La muestra consta de 2.418 posts, publicados entre abril y mayo de 2020 y enero y febrero de 2021. Independientemente de las profundas desigualdades socioeconómicas de la región, ésta presenta un intenso uso de Internet y de las redes sociales. Los resultados señalan a Instagram como una herramienta de comunicación emergente para las autoridades de los países analizados, que la utilizan con diferentes enfoques. En la muestra predominaron los mensajes centrados en la prevención, la promoción de la salud y las instituciones. El card fue el formato dominante para la presentación de los posts. La pandemia de COVID-19 fue el tema más frecuente. En las consideraciones finales, hay sugerencias de buenas prácticas dirigidas a los perfiles evaluados, basadas en las cuestiones presentadas en el análisis.

Palabras clave: salud pública, redes sociales, Instagram, América del Sur, autoridades sanitarias. published between April and May 2020 and January and February 2021. Regardless of the region's deep socio-economic inequalities, it presents an intense use of the Internet and social media. The results pointed to Instagram as an emerging communication tool for the analyzed countries' authorities, which used it with different approaches. In the sample, messages that focused on prevention, health promotion, and institutions predominated. The card was the dominant language for presenting the posts. The COVID-19 pandemic was the most frequent topic. In the final considerations, there are good practices suggestions directed to the evaluated profiles, based on the issues presented in the analysis.

Keywords: public health, social media, Instagram, South America, health authorities.

\section{INTRODUCCIÓN}

A pesar de que Instagram es considerada como una herramienta estratégica en el contexto de la salud pública (Fung et al., 2019; Guidry et al., 2019; Kamel Boulos, Giustini, y Wheeler, 2016; Muralidhara y Paul, 2018; Sendra-Toset y Farré-Coma, 2016; Soares, Recuero, Viegas, Bonoto, y Hüttner, 2021; Teoh et al., 2019; O’Donnell y Willoughby, 2017), existen pocos estudios referentes al uso de esta aplicación en los países en desarrollo y en aquellos de ingresos bajos, por consiguiente, son escasos los trabajos que hacen la comparación entre estos países (Novillo-Ortiz y Hernández-Pérez, 2017; Tursunbayeva, Franco, y Pagliari, 2017; Lwin, Lu, Sheldenkar, y Schulz, 2018; Pinto, Antunes, y Almeida, 2021). Una revisión sistemática sobre el uso de esta aplicación ( $a p p$ ) en el ámbito de la salud pública, identifica Instagram como una herramienta de promoción de la salud que tiene la capacidad de envolver a los usuarios; con potencial de impacto en aspectos relacionados con la salud en la sociedad contemporánea, relativamente por su atractivo aspecto visual (Pinto, Antunes, y Almeida, 2020). Entre el 2015 y 2021, se ha visto el crecimiento de estudios afines sobre esta app, en los cuales la plataforma dejó de ser analizada en conjunto con otras redes sociales y pasó a ser evaluada individualmente. Estos estudios fueron desarrollados en el contexto de la salud, la tecnología y la computación, así como también en el área de las ciencias sociales. En este período predominaron análisis de casos puntuales, observados a corto plazo en países desarrollados.

En esta investigación se optó por estudiar el uso de Instagram por los Ministerios de la Salud de los países de América del Sur y se pretende destacar las estrategias adoptadas para aumentar la información sobre salud. Hay que tener en cuenta que en este estudio se integra el escenario de América Latina y el Caribe, marcado por profundas desigualdades 
socioeconómicas, que impactan directamente el acceso a la salud (Organización Panamericana de la Salud, 2020), pero con un intenso uso del Internet y de las redes sociales. No obstante, a pesar del notorio desequilibrio socioeconómico, esta región presentó un crecimiento significativo en el número de usuarios de Internet. En los últimos cinco años, la penetración de Internet en América Latina pasó del 50,0 \% para un $70 \%$, con 130 millones de nuevos usuarios (Echeberría, 2020). La proporción de usuarios de Internet presentes en redes sociales osciló entre 83,5 \% en Chile y $49 \%$ en Venezuela (Statista, 2021). En este intervalo, la adhesión de las autoridades de salud de América del Sur a Instagram fue de forma creciente, pasando de tres perfiles ( $25 \%$ de los países) activos, hasta el 2015 , para nueve perfiles (75 \%) en el 2019. Sólo los gobiernos de Guyana, Ecuador y Surinam no tenían perfil en Instagram a lo largo de esta investigación. Esta app, es usada por entidades de salud desde el 2011, con la creación del perfil de la Organización Mundial de la Salud (OMS), seguidamente en el año 2012 la Organización Panamericana de la Salud (OPS) inició su perfil. En la muestra analizada, Brasil fue el primer país a unirse a Instagram en el 2013 y Argentina fue el último en el año 2019.

Este crecimiento coincidió con la expansión de Instagram, el cual cumplió una década en el 2020. El enfoque de Instagram de compartir visualmente imágenes está presente en el día a día de más de 1 billón de usuarios; es considerado estratégico para observar interacciones sociales, mediadas por imágenes, que pueden revelar diversos aspectos socioculturales (Manovich, 2017; Statista, 2019; Alcolea, Rodríguez, y Núñez, 2020); de igual forma, se destaca como una de las principales plataformas de redes sociales entre jóvenes adultos, conectados por dispositivos móviles en áreas urbanas. En el 2012, Instagram fue comprado por Facebook y comenzó a compartir contenidos con las aplicaciones de esa plataforma (Frier, 2020). Esa transición amplió el uso de la comunicación visual como vector de creación y mantenimiento de interacciones sociales (Serafinelli, 2018), así como también, cercó el uso de esta herramienta a la publicidad de marcas. Instagram pasó de ser una aplicación para compartir imágenes, para una plataforma de medición de contenidos, en la cual los posts personales pasaron a dividir el espacio con publicidad de empresas, gobiernos y de influencers.

Es importante destacar que Instagram es una plataforma relevante para analizar la salud pública, teniendo en cuenta la presencia de los ciudadanos, de las autoridades de salud, de los profesionales y de otros actores relacionados con este tema. El estudio de We Are SocialHootsuite (We Are Social, 2021) sobre los hábitos globales de los usuarios en la red, identificó la búsqueda de información como el principal motivo del uso de Internet (63\%) en el 2021. La búsqueda en la red por informaciones y productos de salud representa un 36,3\% del uso de Internet. Las redes sociales fueron señaladas por el $45 \%$ de los usuarios del estudio, como el canal para obtener informaciones sobre productos y servicios de interés para consumir. En este sentido son consideradas un punto de observación relevante para la salud pública, ya sea desde el punto de vista de las prácticas de sus actores, como de la visibilidad de los temas de la salud en estas plataformas con determinados intereses económicos.

Este trabajo busca responder a la pregunta: ¿Cómo las autoridades sanitarias de América del Sur utilizan Instagram de manera que sea herramienta de salud pública?; y tiene como objeto analizar los perfiles de los Ministerios de la Salud de América del Sur en Instagram. Se trata de un estudio exploratorio anclado en la revisión de literatura y el análisis de contenido, sobre un tema dinámico y que esta en pleno auge. La muestra es compuesta por 2.418 posts, publicados 
por nueve perfiles (Argentina, Bolivia, Brasil, Chile, Colombia, Paraguay, Perú, Uruguay y Venezuela), entre abril - mayo del 2020 y enero - febrero del 2021.

Los resultados apuntaron diferentes usos de la plataforma por parte de las autoridades sanitarias, contemplando los intereses de los ciudadanos y de las instituciones, en el ámbito de la salud pública. La pandemia del COVID-19 promovió la producción continua de contenidos en los perfiles e impactó directamente las temáticas abordadas. Predominaron mensajes orientados a la prevención, la promoción de la salud (PS) y a las instituciones. El lenguaje dominante para la presentación de los posts fue el card (texto e imagen). En las consideraciones finales fueron hechas sugerencias de las buenas prácticas a los perfiles evaluados, a partir de las interrogantes presentadas en el análisis.

\section{SALUD PÚBLICA EN INSTAGRAM}

\subsection{Marco teórico}

La presencia de las autoridades sanitarias en Instagram puede aproximarlas a sus stakeholders, favorecer la difusión de contenidos segmentados según el público; orientar sobre estrategias de prevención y sobre el funcionamiento de las organizaciones sanitarias (Guidry, Jin, Orr, Messner, y Meganck, 2017; Seltzer, Jean, Kramer-Golinkoff, Asch, y Merchant, 2015; Lwin et al., 2018; Tang, Bie, Park, \& Zhi, 2018). Esta app también permite la actualización de las informaciones con agilidad y la monitorización de las estrategias adoptadas (Moorhead et al., 2013; Filimonov, Russmann, y Svensson, 2016; Mesquita, Ruão, y Andrade, 2020). Por medio de estas plataformas las autoridades pueden reducir las incertidumbres, tranquilizar al público y estimular la confianza en los representantes de la salud pública, sobre todo en una crisis sanitaria (Eriksson, 2018; Viswanath, Lee, y Pinnamaneni, 2020). Según Seltzer et al. (2015) las redes sociales ofrecen una ventana para el conocimiento, sentimiento y comportamiento público, así como también son un canal de comunicación para mantener y promover la salud. Otra razón para reiterar la necesidad de esta presencia digital es el creciente avance de tópicos nocivos para la salud en la plataforma (Kamel Boulos et al., 2016) - p. ej. la industria de fastfood (Laestadius y Wahl, 2017; Vassallo et al., 2018; Klassen, Borleis, Brennan, Reid, McCaffrey, y Lim, 2018) y de sustancias ilícitas (Cherian, Westbrook, Ramo, y Sarkar, 2018; Hendriks, Wilmsen, van Dalen, y Gebhardt, 2020).

La actuación de las autoridades sanitarias durante la pandemia del COVID-19 mostró el protagonismo de los actores públicos para afrontar esta crisis global en Instagram. La plataforma fue utilizada durante el 2020 por las agencias de salud en escala internacional y nacional como la OMS, la OPS y las autoridades sanitarias de Portugal, Brasil y España (VillegasTripiana, Villalba-Diaz, y López-Villegas, 2020; Pinto, Brasileiro, Antunes, y Almedia, 2020). Se ha averiguado que los contenidos basados en imágenes adoptaron un abordaje de la comunicación de riesgo (Glik, 2007; World Health Organization, 2018) para orientar sobre la prevención del virus, la PS, la vacunación, también para estimular la adopción de medidas de seguridad, ampliar la confianza en los actores de la salud pública y para combatir la desinformación sobre la pandemia (Eysenbach, 2020; Limaye et al., 2020; Martin, Karafillakis, Preet, y Wilder-Smith et al., 2020). Desde el 24 de marzo del 2020, Instagram ha encaminado a los usuarios que realizan búsqueda sobre COVID-19, para el perfil de la OMS, designándolo como la autoridad central para difundir informaciones sobre la pandemia en el contexto de la infodemia en curso. 
En el primer año de la crisis sanitaria, el perfil de la OMS pasó de 2,6 millones de seguidores (marzo de 2020) para 9,9 millones en marzo de 2021. Pinto, Brasileiro, Antunes, y Almedia (2020) mapearon los primeros 100 días del abordaje del COVID-19 en Instagram, del cual identificaron que la OMS ejerció influencia sobre los usuarios, por medio de la oferta de contenido basado en evidencias científicas, sin embargo, el perfil no respondió ninguna de las preguntas hechas en los comentarios. Este ejemplo refleja una práctica recurrente sobre las posibilidades de interacción entre actores de la salud pública y sus stakeholders en Instagram. Estudios demuestran que las entidades de salud tienen dificultad en garantizar una perspectiva participativa en relación con los ciudadanos que integran estas redes sociales, quienes han sido subestimados por estos actores, que adoptan perspectivas unidireccionales, con énfasis en contenido institucional (Galiano-Coronil y MierTerán-Franco, 2019; Gesser-Edelsburg, Diamant, Hijazi, y Mesch, 2018; Guidry et al., 2019; Lwin et al., 2018; Miola y Marques, 2020; Ramanadhan, Mendez, Rao, y Viswanath, 2013; Silva, Aragão, y Pretto, 2021). Esta falta de diálogo limita la actuación de las autoridades en el escenario de la crisis sanitaria, en el cual la población precisa de ser incluida como parte central en las decisiones para enfrentar la crisis (World Health Organization, 2018).

Entre las buenas prácticas para el uso de Instagram en el ámbito de la salud pública, el diálogo con los ciudadanos se destaca como la acción capaz de dar el potencial y el alcance de los canales en la plataforma (Erikson, 2018; Galiano-Coronil y MierTerán-Franco, 2019; GesserEdelsburg et al., 2018; Lwin et al., 2018). Teoh et al. (2019) sugieren que las estrategias de aproximación al público sean adaptadas a la edad del usuario, considerando los diferentes orígenes (socioeconómicos), con diversidad de mensajes (incluyendo diferentes tipos de imágenes - p. ej. contenido de humor e infografía), en el uso de diferentes redes sociales. Santarossa y Woodruff (2018) recomiendan el uso de líderes de opinión (enfoque semejante a la de los digital influencers) para diseminar los contenidos sobre salud y así es posible tener más público para participar en las campañas de salud. Tang et al. (2018) orientan el uso de portavoces alternativos como fuentes de información (médicos, investigadores, etc.) para difundir con éxito informaciones útiles y promover la prevención y tratamiento en el momento oportuno, sobre todo cuando hay escenarios de desconfianza en los entes gubernamentales. Novillo-Ortiz y Hernández-Pérez (2017) sugieren una adecuación del vocabulario para referirse a términos relacionados con la salud pública, facilitando así la comprensión de las informaciones. Guidry et al. (2017) proponen que las organizaciones de salud pública deban enviar mensajes más positivos (basados en soluciones), en vez de mensajes negativos durante la crisis sanitaria. Rudd y Baur (2020) sostienen que las estrategias de comunicación de los actores de la salud pública deben incluir informaciones y recursos para ampliar los conocimientos sanitarios de todos, especialmente de los más vulnerables. Según los autores, los mensajes deben ser claros y alcanzables, incluso para quienes viven en contextos de desigualdad.

Cabe destacar otro aspecto importante a ser considerado en la aproximación entre la salud pública e Instagram, es la tensión entre el interés público y el privado. Instagram es una plataforma online, que funciona a partir de la producción y el intercambio de datos de usuarios, con perfiles identificables, con lógicas comerciales ancoradas en el compromiso de los usuarios; él impacta en al reorganización de las relaciones interpersonales, en el consumo de bienes y discusiones de la sociedad contemporánea (d’Andréa, 2020; Soares et al., 2021); y 
es propiedad de Facebook, una de las Big Five (Alphabet-Google, Amazon, Apple, Facebook y Microsoft) - empresas de tecnología que controlan parte del mercado digital. Este grupo es responsable por administrar un ecosistema opaco y complejo en el cual las conexiones son invisibles al público y difíciles de controlar por parte de la sociedad (van Dijck, Nieborg, y Poell, 2019). Desde el 2016, Instagram incluyó los algoritmos como elemento para determinar lo que es relevante para cada usuario, a partir del direccionamiento de flujos de contenidos. Esta lógica opaca afecta la visibilidad de contenidos de interés público, como los de la salud, y perturba el propio derecho a la comunicación (Manovich, 2017; Silva et al., 2021). Al reflexionar sobre las interferencias de la lógica del algoritmo de Instagram, en los perfiles de las autoridades de salud pública, este artículo concuerda con Bucher (2018) y Lemos y Pastor (2020), demostrando la importancia de investigar como estos dispositivos se vuelven relevantes en situaciones específicas, en el caso de la salud en las redes sociales. Este estudio destaca la influencia de los algoritmos en los perfiles de la salud, pero no los analiza.

\subsection{Objetivos, hipótesis y metodología}

El objetivo principal es identificar los perfiles de Instagram de las autoridades sanitarias en los 12 países de América del Sur (Argentina, Bolivia, Brasil, Chile, Colombia, Ecuador, Guyana, Paraguay, Perú, Surinam, Uruguay y Venezuela) y hacer un análisis cualitativa y cuantitativa sobre los usos de esta plataforma, para ampliar las informaciones relacionadas con la salud pública.

Los objetivos específicos son analizar el contenido de los perfiles de Instagram de los Ministerios de la Salud de países de América del Sur para exponer las principales estrategias de utilización de cada perfil y elaborar directrices con buenas prácticas para la gestión de los contenidos en los perfiles, con el propósito de ampliar el alcance de estos canales.

La primera hipótesis es que Instagram ha sido utilizado para ampliar información en salud, pero hay una baja interacción entre las autoridades y los usuarios de la plataforma y la segunda es que las autoridades de salud adoptaron Instagram como herramienta institucional, en detrimento del contenido sobre salud.

El diseño metodológico de este estudio fue desarrollado en dos partes. La primera correspondió a la revisión de literatura sobre los ejes de Comunicación y salud, el uso de las tecnologías de la información y la comunicación por parte de las autoridades de salud pública (ehealth) y el uso de plataformas digitales en salud; y la segunda envolvió un estudio exploratorio compuesto por el análisis de contenido (Bardin, 2011) de los nueve perfiles entre el 2020 y el 2021. Los indicadores de este análisis de contenido se dividieron en: a) contexto, b) tema, c) contenido, y d) interacción.

a) Contexto: se refiere al uso de las herramientas disponibles en la plataforma por las autoridades (feed, stories, IGTV, etc.), la fecha de creación del perfil y el flujo de publicaciones hasta marzo de 2021.

b) Tema: señala la temática contenida en los posts y las estrategias adoptadas para abordar la salud pública. Los posts se clasificaron en dos grupos temáticos: salud (publicación que no aborda la pandemia) y COVID-19 (contenido que aborda la pandemia). Esta división fue necesaria por el volumen de publicaciones sobre la pandemia. Así mismo, estos dos grupos de posts se clasificaron con las subcategorías 
relacionadas con las estrategias: prevención (de enfermedades), promoción de la salud (con indicaciones de práctica de actividad física, alimentación saludable, etc.), institucional (contenido sobre el Ministerio de Sanidad y el gobierno), celebridades (para difundir mensajes de perfil), y fechas sanitarias (establecidas por la OMS y las autoridades sanitarias nacionales). Estas subcategorías ya fueron validadas en un estudio anterior (Pinto et al., 2020). Además, se realizó una evaluación previa con el perfil del gobierno de Chile, a partir de la cual se observó la necesidad de insertar las subcategorías temáticas accountability (transparencia de las acciones del gobierno) y vacuna.

c) Contenido: se observa el tipo de publicación (card, fotografía o video), sus leyendas e imágenes y el público citado en los posts (general, neutro e infantil).

d) Interacción: son evaluados los indicadores de audiencia (número de seguidores, likes (me gusta), comentarios y visualizaciones) de los perfiles. A partir de estos datos, se puede obtener indicios de las actividades de los mismos. Los indicadores de audiencia son valores que ubican el alcance de los mensajes, pero no son precisos, pues son influenciados directamente por los algoritmos del propio Instagram - cuya lógica no es descrita por la app.

La muestra está compuesta por 2.418 publicaciones, recogidas en nueve perfiles y tendrán aspectos cuantitativos y cualitativos observados en función de los indicadores mencionados anteriormente. Se optó por analizar el número total de publicaciones realizadas por los perfiles durante un período de cuatro meses. Delimitándose los meses de abril y mayo del 2020, y los meses de enero y febrero del 2021. Abril del 2020 fue el primer mes de la muestra, en el cual todos los perfiles tenían publicaciones. Dentro de este intervalo es tomado como referencia de inicio de la pandemia en la región y el período en el que Sudamérica se convirtió en uno de los epicentros del COVID-19. Los datos de este estudio fueron extraídos con el software Instabro (publicaciones, leyendas e indicadores de audiencia). Para la clasificación posterior de los posts los mismos fueron realizados de forma manual por las investigadoras y posteriormente introdujeron los datos en hojas de cálculo para su análisis.

Esta estrategia metodológica se basa en datos expuestos al público y no tuvo acceso a las informaciones de alcance de los mensajes (direccionados con precisión apenas a los gestores de los perfiles), con relación a las estrategias de acción delineadas por cada Ministerio de la Salud. En la actualidad, aún no se ha podido comprobar si los perfiles utilizaban acciones artificiales para potenciar el alcance de los contenidos. Con todo, se defiende la pertinencia de este abordaje descriptivo, considerando la escasez de investigadores sobre el uso de Instagram en el entorno de la salud en América del Sur, en contrapartida al expresivo avance de la plataforma en la región.

\section{RESULTADOS}

Instagram es utilizado como herramienta de comunicación sobre temas de salud pública por nueve autoridades de salud en América del Sur. La tabla 1 presenta un resumen de los perfiles.

Tabla 1

Resumen de los perfiles 


\begin{tabular}{|c|c|c|c|}
\hline País/ Perfil & Fecha de creación & Datos Demográficos & Resumen del Perfil \\
\hline $\begin{array}{l}\text { Argentina } \\
@ \text { @salnacion }\end{array}$ & 2019 & $\begin{array}{l}\text { Población: } 45 \text { millones } \\
\text { Usuarios de Internet conectados } \\
\text { a las redes sociales (UIRS): 79,3\% }\end{array}$ & $\begin{array}{l}471.910 \text { seguidores } \\
1.008 \text { posts / } 7 \text { stories } \\
269 \text { IGTV }\end{array}$ \\
\hline $\begin{array}{l}\text { Bolivia } \\
\text { @saluddeportesbo }\end{array}$ & 2015 & $\begin{array}{l}\text { Población: } 11 \text { millones } \\
\text { UIRS: } 69,8 \%\end{array}$ & $\begin{array}{l}8.071 \text { seguidores } \\
1.177 \text { posts / } 5 \text { stories }\end{array}$ \\
\hline $\begin{array}{l}\text { Brasil } \\
\text { @minsaude }\end{array}$ & 2013 & $\begin{array}{l}\text { Población: } 213 \text { millones } \\
\text { UIRS: 70,3\% }\end{array}$ & $\begin{array}{l}2,8 \text { millones seguidores } \\
8.116 \text { posts / } 404 \text { IGTV } \\
5 \text { reels }\end{array}$ \\
\hline $\begin{array}{l}\text { Colombia } \\
@ \text { minsaludcol }\end{array}$ & 2015 & $\begin{array}{l}\text { Población: } 51 \text { millones } \\
\text { UIRS: } 76,4 \%\end{array}$ & $\begin{array}{l}422.199 \text { seguidores } \\
1.549 \text { posts / } 3 \text { stories } \\
151 \text { IGTV }\end{array}$ \\
\hline $\begin{array}{l}\text { Chile } \\
@ \text { ministeriosalud }\end{array}$ & 2016 & $\begin{array}{l}\text { Población: } 19 \text { millones } \\
\text { UIRS: } 83,5 \%\end{array}$ & $\begin{array}{l}343.496 \text { seguidores } \\
1.266 \text { posts / } 3 \text { stories } \\
6 \text { IGTV }\end{array}$ \\
\hline $\begin{array}{l}\text { Paraguay } \\
\text { @msaludpy }\end{array}$ & 2019 & $\begin{array}{l}\text { Población: } 7 \text { millones } \\
\text { UIRS: } 59,9 \%\end{array}$ & $\begin{array}{l}277.016 \text { seguidores: } \\
3.124 \text { posts / } 15 \text { stories } \\
99 \text { IGTV }\end{array}$ \\
\hline $\begin{array}{l}\text { Perú } \\
\text { @minsa_peru }\end{array}$ & 2018 & $\begin{array}{l}\text { Población: } 33 \text { millones } \\
\text { UIRS: } 81,4 \%\end{array}$ & $\begin{array}{l}537.625 \text { seguidores } \\
1.491 \text { posts / } 6 \text { stories } \\
289 \text { IGTV }\end{array}$ \\
\hline $\begin{array}{l}\text { Uruguay } \\
\text { @mspuruguay }\end{array}$ & 2018 & $\begin{array}{l}\text { Población: } 3 \text { millones } \\
\text { UIRS: } 83,3 \%\end{array}$ & $\begin{array}{l}97.040 \text { seguidores } \\
968 \text { posts / } 1 \text { story } \\
27 \text { IGTV }\end{array}$ \\
\hline $\begin{array}{l}\text { Venezuela } \\
\text { @mppsalud_vzla }\end{array}$ & 2016 & $\begin{array}{l}\text { Población: } 28 \text { millones } \\
\text { UIRS: } 49,0 \%\end{array}$ & $\begin{array}{l}14.107 \text { seguidores } \\
1.766 \text { posts / } 2 \text { stories } \\
36 \text { IGTV / } 5 \text { reels }\end{array}$ \\
\hline
\end{tabular}

Fuentes: (CEPAL, 2021; Statista, 2021). Elaboración propia.

Partiendo de la información de la tabla 1, refleja el uso de Instagram por parte de las autoridades sanitarias en América del Sur de países como Brasil, Perú, Paraguay y Argentina manteniendo un flujo de posts desde la creación de los perfiles. Cabe destacar que en lo resto el flujo de publicaciones fue irregular, con intervalos de la no producción de contenidos. Desde que se inició la crisis del COVID-19, los perfiles pasaron a publicar con frecuencia en Instagram, 
siendo Venezuela y Brasil los países que disminuyeron en número de posts publicados durante enero del 2020 y febrero del 2021. Además del feed de posts, los países utilizaran los stories para destacar temas y la IGTV para almacenar videos. De igual manera, los vídeos de tipo reels, de hasta 30 segundos de duración, sólo han sido utilizados en Brasil y Venezuela. Este tipo de herramienta se creó en 2020 para hacer frente a la expansión de la app Tik Tok. La Figura 1 reúne los indicadores de un ano de posts:

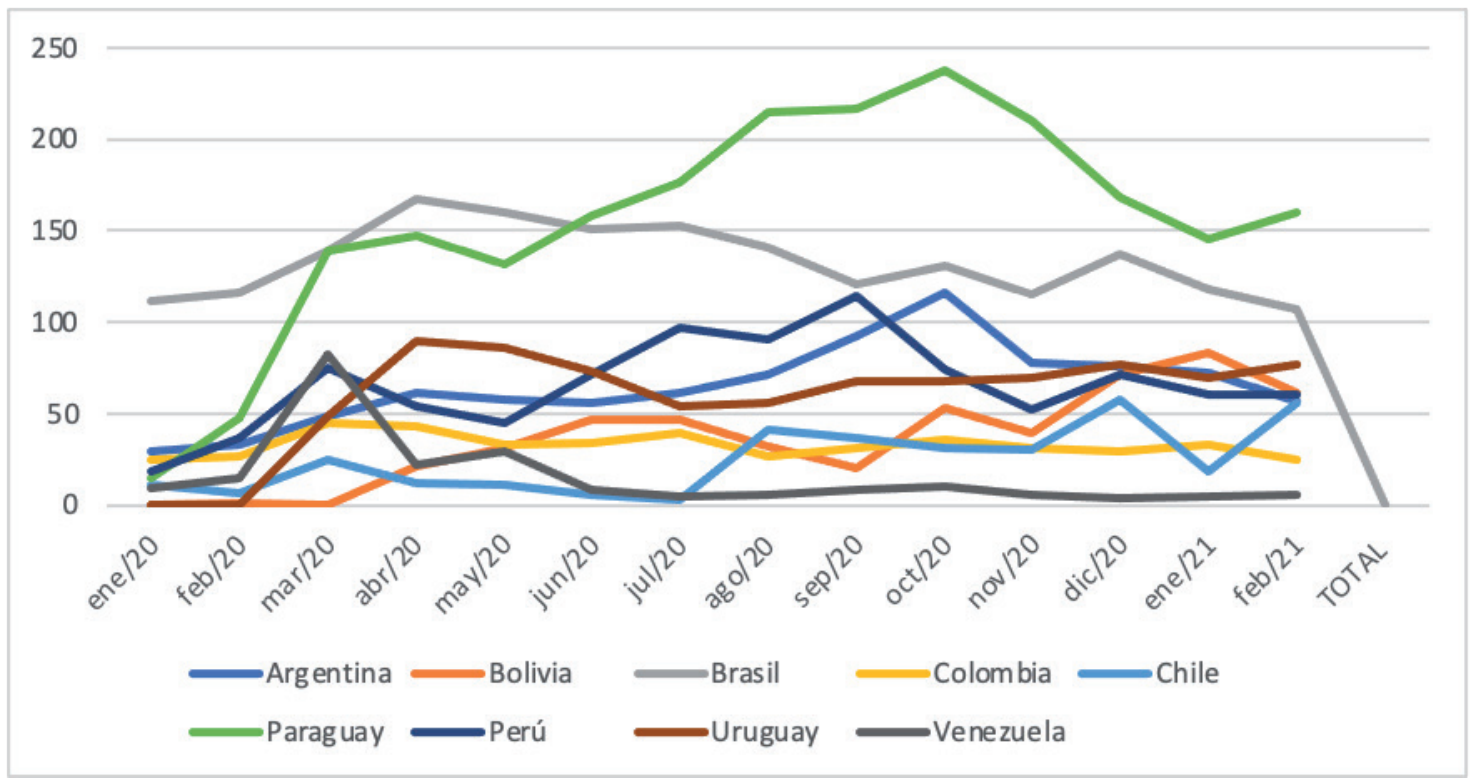

Figura 1. Posts de países entre 2020 y 2021

Fuente: elaboración propia.

Observando los posts de los países entre el 2020 y 2021 los mismos fueron divididos en dos grupos: un con los posts de la salud que no citaron la pandemia y otro con los posts sobre el COVID-19. La crisis sanitaria fue el principal tema de los perfiles, variando de un $67,7 \%$ de los posts en Venezuela y $91,8 \%$ en Bolivia. Los temas de salud abordados fueron de fechas relacionadas con días conmemorativos de la salud, PS, acciones institucionales y posts sobre prevención. Además del COVID-19, entre el 2020 y el 2021, los perfiles relataban la presencia de brotes de Dengue; únicamente Venezuela y Uruguay destacaron temas de la salud entre sus estrategias centrales, en donde fueron encontrados publicaciones de fechas de salud (Venezuela) y de vacunación de H1N1 (Uruguay). La prevención fue el principal tema abordado referente al COVID-19, siendo dominante en: Colombia (41,3\%), Chile (31,9\%), Perú (26\%), Bolivia $(24,4 \%)$ y Argentina $(22,9 \%)$. Brasil presentó el menor espacio en orientar a la población en como prevenir el virus (6,4 \%). La accountability fue la principal estrategia de Brasil (38,6 \%), Uruguay (38,3 \%) y Paraguay (37,1 \%), destacando las acciones de enfrentamiento de la pandemia; además los mensajes institucionales figuraron entre los temas principales de siete de los países de la muestra, siendo Venezuela ( $29 \%$ ) el país que más predominó con este tipo de contenido. La tabla 2 presenta un resumen de los temas dominantes: 
Tabla 2

Resumen de los temas dominantes

\begin{tabular}{|c|c|c|}
\hline País & Posts (total) & Temas dominantes \\
\hline Argentina & 248 & $\begin{array}{l}\text { Prevención (COVID-19) - 22,9 \% } \\
\text { Accountability (COVID-19) - 18,5\% } \\
\text { Institucional (COVID-19) - 17,7 \% }\end{array}$ \\
\hline Bolivia & 196 & $\begin{array}{l}\text { Prevención (COVID-19) - 24,4 \% } \\
\text { PS (COVID-19) - 20,9\% } \\
\text { Institucional (COVID-19) - 18,8\% }\end{array}$ \\
\hline Brasil & 556 & $\begin{array}{l}\text { Accountability (COVID-19) - 38,6 \% } \\
\text { Institucional (COVID-19) - 20,6\% } \\
\text { PS (COVID-19) - 10,6\% }\end{array}$ \\
\hline Colombia & 133 & $\begin{array}{l}\text { Prevención (COVID-19) - 41,3 \% } \\
\text { PS (COVID-19) - } 27 \% \\
\text { Institucional (COVID-19) - } 15 \%\end{array}$ \\
\hline Chile & 97 & $\begin{array}{l}\text { Prevención (COVID-19) - 31,9\% } \\
\text { Institucional (COVID-19) - 26,8\% } \\
\text { Vacuna (COVID-19) - 20,6 \% }\end{array}$ \\
\hline Paraguay & 584 & $\begin{array}{l}\text { Accountability (COVID-19) - 37,1 \% } \\
\text { Prevención (COVID - 19) - 15,4 \% } \\
\text { Institucional (COVID-19) - 15,2\% }\end{array}$ \\
\hline Perú & 219 & $\begin{array}{l}\text { Prevención (COVID-19) - } 26 \% \\
\text { PS (COVID-19) - } 21 \% \\
\text { Institucional (COVID-19) - 18,2 \% }\end{array}$ \\
\hline Uruguay & 323 & $\begin{array}{l}\text { Accountability (COVID-19) - 38,3\% } \\
\text { PS (Salud General) - 22,2 \% } \\
\text { PS (COVID-19) - } 12 \%\end{array}$ \\
\hline Venezuela & 62 & Institucional (COVID-19) - $29 \%$ \\
\hline
\end{tabular}




\begin{tabular}{|l|l|l|}
\hline & & Accountability (COVID-19) $-27 \%$ \\
& Fechas de la Salud (Salud General) - 16,1 \% \\
\hline
\end{tabular}

Fuente: elaboración propia.

Al respecto de la tabla 2, en cuanto a las medidas preventivas sobre el COVID-19 enfatizaron las orientaciones de la OMS en cuanto a la etiqueta respiratoria, la higiene personal y el uso de las mascarillas. Argentina utilizó especialistas para abordar el tema y destacar los cuidados para diferentes segmentos (personas con VIH, autistas, etc.) y divulgó campañas educativas por videos. En Colombia los mensajes también orientaron a los trabajadores informales. Las desigualdades económicas fueron contempladas en pocos posts de la muestra. En Perú se enseñó como producir un desinfectante accesible a base de lejía y explicó que este tipo de sustancia no debe ser usada en el cuerpo, solo en las superficies. La producción de las mascarillas caseras fue orientada en el 2020 en Brasil, Chile, Perú y Colombia, debido a la escasez inicial de equipamientos de protección individual. Perú y Paraguay tradujeron orientaciones para idiomas originarios y Venezuela y Uruguay incluyeron lenguaje de señas en videos. Las orientaciones fueron direccionadas a los adultos, con limitado espacio para niños.

Es así como, en los posts de PS relacionados a la pandemia fueron divulgados canales digitales de atendimiento, canales oficiales para información, además de las orientaciones sobre alimentación saludable, vacunación y prácticas de actividad física. En Bolivia fue hecha una campaña para fomentar la alimentación saludable, como forma de protección contra el virus, con todo algunos posts no aclararon que el consumo de estos alimentos debería estar acompañado de medidas de prevención contra el virus. Es así como, en Paraguay los mensajes reforzaban el estímulo a nuevas prácticas culturales, al orientar sobre el no compartir los utensilios en el consumo de bebidas, que culturalmente son consumidas de modo compartido. En ese mismo contexto, el gobierno Uruguayo destacó también el entrenamiento de profesionales en ambientes digitales y recomendó a la población a no usar la ivermectina para la prevención del COVID-19. En los perfiles de Brasil y Venezuela hubo apoyo al uso de cloroquina, cuando la OMS y la OPS ya se habían opuesto (Organización Panamericana de Salud, 2020). El abordaje institucional en el contexto de la crisis sanitaria apuntó a una principal diferencia de estrategias en la muestra. En cuanto Argentina, Chile, Colombia, Perú y Uruguay priorizaron el interés público de los ciudadanos, los perfiles de Bolivia, Brasil, Paraguay y Venezuela favorecieron la visibilidad del gobierno. Hubo intensa personalización del contenido institucional con publicaciones sobre los ministros de parte de: Paraguay $(15,9$ $\%)$, Argentina (14,5\%) y Brasil $(9,3 \%)$.

En cuanto al indicador de contenido, se observó que el lenguaje dominante para presentar los posts fue el card, con la mayor presencia en Bolivia $(98,4 \%)$ y la menor $(37,9 \%)$ en Argentina el único perfil con el predominio de videos en la muestra. El uso dominante de las fotografías fue verificado en Venezuela $(61,2 \%)$ y Paraguay $(40,2 \%)$. Mientras que los cards del tipo texto fueron adoptados principalmente en: Brasil (56,2\%), Uruguay $(53,5 \%)$ y Chile $(49,4 \%)$. Los cards teniendo ilustraciones fueron dominantes en Colombia $(42,1 \%$ y Perú $(33,3 \%)$. En toda la muestra, predominan los contenidos neutros, sin identificación de género. Colombia fue el país con la mayor proporción de imágenes, incluyendo hombres y mujeres $(34,5 \%)$. Venezuela destacó la mayor proporción de posts envolviendo apenas hombres (25,8\%). Las mujeres tuvieron más visibilidad en el feed de Bolivia (19,8 \%). Las leyendas complementaron las 
imágenes e indicaron los sitios web de los gobiernos para profundizar temas en los perfiles de Argentina, Brasil, Chile, Colombia y Perú. Mientras que en Paraguay las leyendas oscilaron entre textos cortos con emojis y textos largos. En Bolivia las leyendas no complementaban las imágenes y usaban términos técnicos con frecuencia y en Uruguay las leyendas tampoco complementaban las imágenes, apenas direccionaban al usuario para conocer las informaciones por un link externo. En Venezuela las leyendas de las imágenes fueron largas semejantes a materias periodísticas. Entre los principales problemas de calidad de contenido identificados en los perfiles analizados, se destacan el exceso de texto en los cards, que implicaban en textos pequeños para lectura. Esta práctica fue frecuente en los perfiles de Perú, Chile, Paraguay y Venezuela; problemas en la edición de las imágenes (la baja calidad de las fotos) fueron identificados en los feeds de Chile, Bolivia, Venezuela y Paraguay. En este último perfil fue recurrente el uso de contenidos impropios al lenguaje de Instagram (p. ej. notas del gobierno y fotos de cirugías).

Por fin, se abordan aspectos del último indicador de este análisis de contenido, denominada la interacción con los indicadores de audiencia ilustrados en la Figura 2. Aunque todos los perfiles analizados presentaron números crecientes de seguidores en el 2020 (Njlitics, 2021), este indicador señala que los perfiles de las autoridades tienen alcance limitado en la población. Los indicadores de audiencia destacan la reducida participación de los usuarios (Ballesteros, 2018), por la baja media de los comentarios y likes. El tipo de interacción dominante estuvo relacionado con el número de visualizaciones de los vídeos. Instagram cuenta esto dato automáticamente, aun cuando el usuario no haya visto hasta final el vídeo en su feed. No requiere una acción activa como un like o un comentario. Los indicadores expuestos aquí apuntan para una mayor interacción en los perfiles que dan prioridad al ciudadano y a las informaciones sobre salud - el perfil brasileño es una excepción.

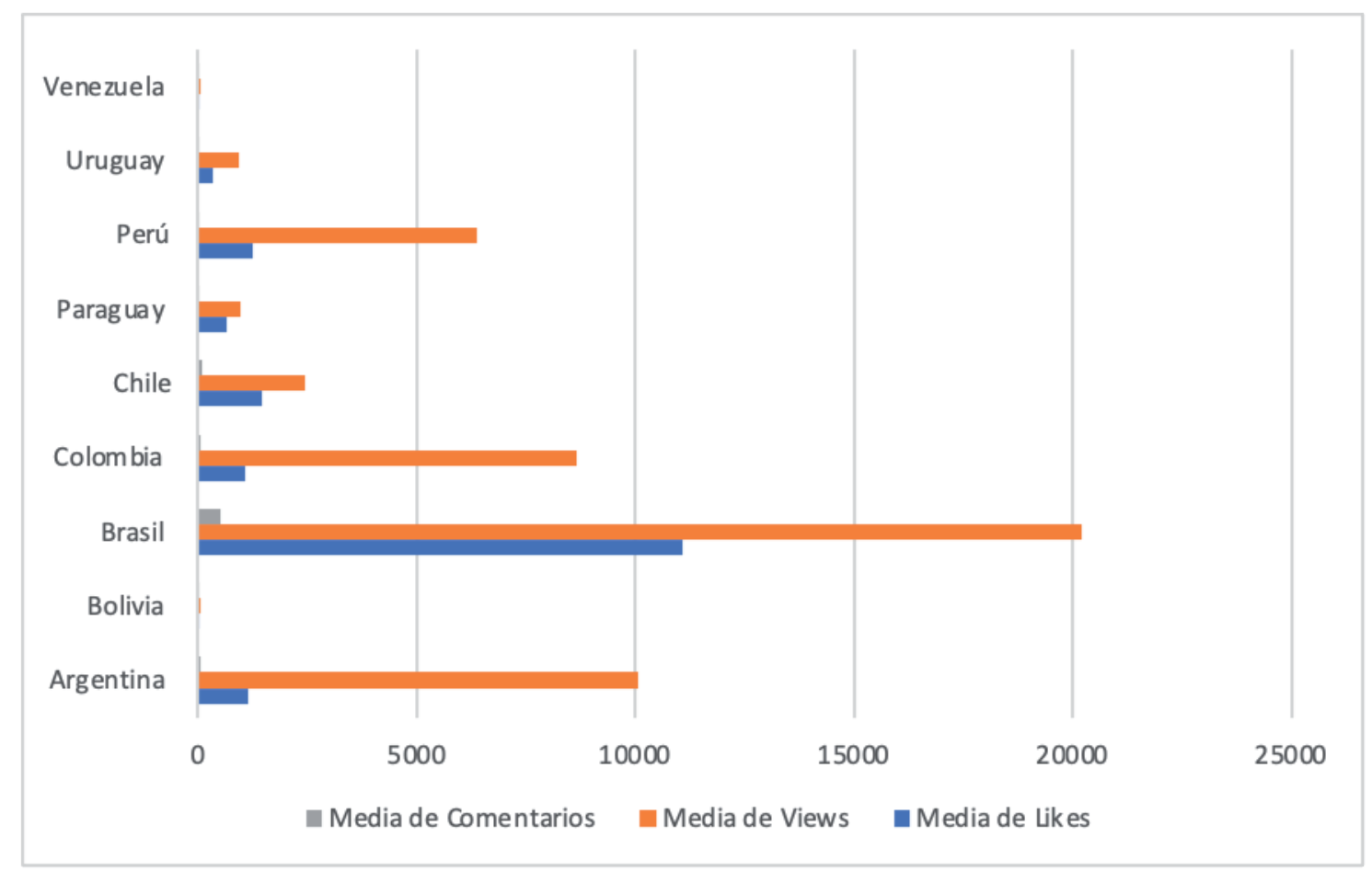

Figura 2. Indicadores de audiencia

Fuente: elaboración propia. 
Por otra parte, los perfiles privilegiaron interlocutores de sus gobiernos (ministros y presidentes), con baja interacción con otras autoridades sanitarias, organismos internacionales y demás stakeholders. En efecto, la misma se produjo específicamente a partir de la citación de entidades en los posts, en los reposts de los stakeholders, en el etiquetado (tagging) de otros perfiles en las publicaciones. Es así, como la OPS fue la entidad externa con mayor destaque en la muestra, con citaciones en los perfiles de Venezuela, Uruguay, Brasil, Bolivia y Paraguay. Ao mismo tiempo, la OMS fue destacada en el contexto de las fechas de la salud; apenas Colombia, Perú y Argentina utilizaron personalidades para dar mayor alcance a los mensajes.

\section{DISCUSIÓN Y CONCLUSIONES}

Los datos de esta investigación apuntan a Instagram como una herramienta emergente de comunicación de las autoridades de salud de nueve gobiernos de América del Sur. Esta red social se suma al Facebook, Twitter y YouTube como fuente de información en salud para los ciudadanos, ofrecidas por las autoridades nacionales en esta región. Estas tres últimas plataformas fueron señaladas por Novillo-Ortiz y Hernández-Pérez (2017) como las principales redes sociales de las autoridades de salud en Latino América. Se cree que el contexto de la pandemia estimuló la mayor adhesión de los países al uso frecuente de la plataforma para ampliar el alcance de sus mensajes sobre salud, actualizar informaciones sobre medidas gubernamentales de contención de la crisis y para difundir campañas educativas. Sin duda se ha comprobado que la urgencia de la pandemia ha tenido un gran impacto en los contenidos de la muestra en los cuatro meses analizados. Los Ministerios contemplaron, en menor proporción, otros temas relevantes en cada país.

Por consiguiente, Instagram fue usado de acuerdo con los intereses de cada gobierno y que el interés público no siempre se demostró ser el tema dominante de los mensajes. En esta muestra apenas Brasil y Venezuela publicaron contenidos desinformativos, relacionados al uso de cloroquina, desaconsejado por la OMS. Eso señala a las propias autoridades sanitarias como productoras de desinformación en el contexto de la infodemia destacada por Eysenbach (2020). Este aspecto podría relacionarse con las tensiones políticas que se viven en los países en desarrollo y exige una mayor investigación comparativa. En los demás países predominaron contenidos sobre prevención y PS, con potencial de ampliar la alfabetización en salud (Rudd y Baur, 2020), teniendo en vista la adaptación parcial de los temas a los contextos socioeconómicos y culturales de los países. Ese estímulo de alfabetización incluyó la divulgación de nuevas formas de atendimiento a la población; orientados sobre el uso de herramientas digitales en el área de la salud (entrenamientos profesionales, apps y teleconsultas). Los mensajes también enfatizaron la importancia de los canales oficiales de comunicación para enfrentar la infodemia. Este contexto, junto con el creciente uso de mensajes de accountability, puede haber estimulado una mayor confianza de la población en las acciones de los gobiernos. Atendiendo las consideraciones de los temas abordados, se observó que los perfiles daban poca relevancia a los aspectos de desigualdad social existentes en los países analizados. Ahora bien, en cuanto al contenido, se observó una baja segmentación de los mensajes de los diferentes perfiles de usuarios existentes en Instagram y el dominio de los posts neutros refleja esta observación. Mientras que los mensajes genéricos, centrados sólo en los adultos (sin delimitar el género, la etnia, grupo de edad, etc.), reducen la posibilidad de dirigir los contenidos a diferentes grupos, con diferentes necesidades de información. 
Se encontraron diferencias significativas, en lo que respecta al contenido transmitido en los países. En Argentina, Brasil, Chile, Colombia y Perú predominaron imágenes de calidad y leyendas coherentes; en Bolivia, Uruguay, Paraguay y Venezuela fueron recurrentes problemas en la calidad de las imágenes y de las leyendas. Este último escenario es similar al presentado por Alcolea et al. (2020) al describir el uso corporativo de Instagram en las universidades privadas españolas en el que no se apreciaba la profesionalización en las publicaciones, especialmente a nivel estético (edición y selección de imágenes adecuadas). Se observó semejanzas en las estrategias de los contenidos en Brasil, Chile y Uruguay, pues los tres optaron por un abordaje textual, en la plataforma cuya atención principal son las imágenes. Solo en Argentina, Chile y Uruguay las interacciones de la muestra recogida fueron menores de lo que en la media general de los perfiles. Lo que puede indicar el interés de la población en ampliar las informaciones durante la crisis sanitaria.

Al considerar Instagram como una herramienta emergente de comunicación entre las autoridades sanitarias y los ciudadanos en los perfiles analizados, con este estudio se sugieren las siguientes recomendaciones de buenas prácticas en el uso de la app: que prioricen a los ciudadanos con contenidos relevantes a su rutina (Lwin et al., 2018); en países en desarrollo o con bajos ingresos, que se utilicen mensajes que tengan en cuenta las desigualdades existentes (Rudd y Baur, 2020); que utilicen líderes de opinión y celebridades para ampliar el alcance de los mensajes, inclusive en escenarios en que hay desconfianza en el gobierno (Santarossa y Woodruff, 2018; Tang et al., 2018); que las leyendas tengan un texto claro y objetivo y términos técnicos más sencillos para optimizar y extender su uso (Novillo-Ortiz y HernándezPérez, 2017); utilizar posts que favorezcan enfoques positivos e informativos (Guidry et al., 2017); consideren el uso de los contenidos de agencias de salud (OMS y OPS), teniendo en vista sus cualidades técnicas; Filimonov et al. (2016) sugieren que los perfiles motivan la movilización de los usuarios, a partir de las especificidades de interacción de la herramienta.

A lo largo de desarrollo del estúdio, las hipótesis del trabajo fueron parcialmente probadas. La hipótesis 1 (Instagram ha sido utilizado para ampliar información en salud, pero hay una baja interacción entre las autoridades y los usuarios de la plataforma) se confirmó con respecto al papel desempeñado por las autoridades sanitarias de América del Sur, entre el 2020 y el 2021, que utilizaron la app para ampliar informaciones en salud, sobre todo en la pandemia del COVID-19. Se notó que los perfiles oficiales dialogan de preferencia con actores del gobierno, en detrimento de agencias internacionales de salud. No fue posible verificar la interacción de los perfiles con los usuarios, a ser mapeada sobre todo en los comentarios. La hipótesis 2 (Las autoridades de salud adoptaron Instagram como herramienta institucional, en detrimento del contenido sobre salud) fue comprobada en los perfiles de Bolivia, Brasil, Paraguay y Venezuela, que priorizaron un abordaje institucional; en los demás países el contenido sobre salud fue dominante.

Para concluir permitieron destacar la importancia de la observación constante del uso de esta app por parte de las autoridades sanitarias en contextos de países en desarrollo, especialmente en Sudamérica, en los que Instagram está en amplia expansión. Se observó el uso de esta plataforma como espacio informativo en países con importante desigualdad de acceso a la salud. Sin embargo, se pude constatar la apropiación de la app con fines políticos entre ellos, la difusión de información errónea de un medicamento que supone un riesgo para la vida de los ciudadanos. Son limitaciones de este estudio la muestra, referente al último año 
publicaciones de los perfiles, la falta de acceso a las estrategias de las autoridades sanitarias para las redes sociales y de datos sobre el alcance de los mensajes. Los estudios futuros deberán considerar las comparaciones longitudinales entre los perfiles para averiguar el uso de Instagram como una herramienta capaz de ampliar la información sobre la salud en contextos de desigualdades.

\section{Referencias}

Alcolea P. M., Rodríguez B. D., y Núñez F. V. (2020). El uso corporativo de Instagram en las universidades privadas españolas. Estudio comparativo de 35 universidades. Ámbitos. Revista Internacional de Comunicación, 47, 109-134. https://doi.org/10.12795/ambitos.2020.i47.06

Ballesteros, C. (2018). El índice de engagement en redes sociales, una medición emergente en la Comunicación académica y organizacional. Razón Y Palabra, 22(January), 96-124. http://revistarazonypalabra.org/index.php/ryp/article/view/1261

Bardin, L. (2011). Análise do Conteúdo - Edição revista e ampliada. Edições 70.

Bucher, T. (2018). If...Then: Algorithmic power and politics. Oxford University Press.

Comisión Económica para América Latina y el Caribe (CEPAL) (2021). Dados Demográficos América do Sul. https://estadisticas.cepal.org/cepalstat/PerfilesNacionales.html?idioma=spanish

Cherian, R., Westbrook, M., Ramo, D., y Sarkar, U. (2018). Representations of Codeine Misuse on Instagram: Content Analysis. JMIR Public Health and Surveillance, 4(1), e22. https://doi.org/10.2196/publichealth.8144

d'Andréa, C. (2020). Pesquisando plataformas online: conceitos e métodos. Editora da Universidade Federal da Bahia (EDUFBA).

Echeberría, R. (2020). Infraestructura de Internet en América Latina: puntos de intercambio de tráfico, redes de distribución de contenido, cables submarinos y centros de datos. CEPAL. https://tinyurl.com/8uttxxst

Eriksson, M. (2018). Lessons for Crisis Communication on Social Media: A Systematic Review of What Research Tells the Practice. International Journal of Strategic Communication, 12(5), 526-551. https://doi.org/10.1080/1553118X.2018.1510405

Eysenbach, G. (2020). How to Fight an Infodemic: The Four Pillars of Infodemic Management The World Health Organization Declares an Infodemic and Crowdsources a Framework. J Med Internet Res, 22(6), 21820. https://doi.org/10.2196/21820

Filimonov, K., Russmann, U., y Svensson, J. (2016). Picturing the Party: Instagram and Party Campaigning in the 2014 Swedish Elections. Social Media and Society, 2(3), $1-11$. https://doi.org/10.1177/2056305116662179

Frier, S. (2020). No Filter: The Inside Story of Instagram. Simon y Schuster.

Fung, I. C. H., Blankenship, E. B., Ahweyevu, J. O., Cooper, L. K., Duke, C. H., Carswell, S. L., Jackson, A. M., Jenkins, J. C., Duncan, E. A., Liang, H., Fu, K. W., y Tse, Z. T. H. (2019). Public Health Implications of Image-Based Social Media: A Systematic Review of Instagram, Pinterest, Tumblr, and Flickr. The Permanente Journal, 24, 1-10. https://doi.org/10.7812/TPP/18.307

Galiano-Coronil, A., y MierTerán-Franco, J. J. (2019). The use of social digital networks by NGDO from a social marketing perspective. Social Sciences, 8(6), 192. https://doi.org/10.3390/SOCSCI8060192

Gesser-Edelsburg, A., Diamant, A., Hijazi, R., y Mesch, G. S. (2018). Correcting misinformation by health organizations during measles outbreaks: A controlled experiment. PLOS ONE, 13(12), e0209505. https://doi.org/10.1371/journal.pone.0209505

Glik, D. C. (2007). Risk communication for public health emergencies. Annual Review of Public Health, 28, 33-54. https://doi.org/10.1146/annurev.publhealth.28.021406.144123

Guidry, J. P. D., Carlyle, K. E., Larose, J. G., Perrin, P., Messner, M., y Ryan, M. (2019). Using the health belief model to analyze Instagram posts about Zika for public health communications. Emerging 
Infectious Diseases, 25(1), 179-180. https://doi.org/10.3201/eid2501.180824

Guidry, J. P. D., Jin, Y., Orr, C. A., Messner, M., y Meganck, S. (2017). Ebola on Instagram and Twitter: How health organizations address the health crisis in their social media engagement. Public Relations Review, 43(3), 477-486. https://doi.org/10.1016/j.pubrev.2017.04.009

Hendriks, H., Wilmsen, D., van Dalen, W., y Gebhardt, W. A. (2020). Picture Me Drinking: Alcohol-Related Posts by Instagram Influencers Popular Among Adolescents and Young Adults. Frontiers in Psychology, 10(January), 1-9. https://doi.org/10.3389/fpsyg.2019.02991

Kamel Boulos, M. N., Giustini, D. M., y Wheeler, S. (2016). Instagram and WhatsApp in health and healthcare: An overview. Future Internet, 8(3), 1-14. https://doi.org/10.3390/fi8030037

Klassen, K. M., Borleis, E. S., Brennan, L., Reid, M., McCaffrey, T. A., y Lim, M. S. (2018). What People "Like": Analysis of Social Media Strategies Used by Food Industry Brands, Lifestyle Brands, and Health Promotion Organizations on Facebook and Instagram. Journal of Medical Internet Research, 20(6), e10227. https://doi.org/10.2196/10227

Laestadius, L. I., y Wahl, M. M. (2017). Mobilizing social media users to become advertisers: Corporate hashtag campaigns as a public health concern. Digital Health, 3, 205520761771080. https://doi.org/10.1177/2055207617710802

Lemos, A., y Pastor, L. (2020). Experiência algorítmica: ação e prática de dado na plataforma Instagram. Revista Contracampo, 39(2), 132-146. https://doi.org/10.22409/contracampo.v0i0.40472

Limaye, R. J., Sauer, M., Ali, J., Bernstein, J., Wahl, B., Barnhill, A., y Labrique, A. (2020). Building trust while influencing online COVID-19 content in the social media world. The Lancet Digital Health, 2(6), e277-e278. https://doi.org/10.1016/S2589-7500(20)30084-4

Lwin, M. O., Lu, J., Sheldenkar, A., y Schulz, P. J. (2018). Strategic uses of Facebook in zika outbreak communication: Implications for the crisis and emergency risk communication model. International Journal of Environmental Research and Public Health, 15(9). https://doi.org/10.3390/ijerph15091974

Manovich, L. (2017) Instagram and contemporary image. http://manovich.net/index.php/projects/instagram-and-contemporary-image

Martin, S., Karafillakis, E., Preet, R., y Wilder-Smith, A. (2020). The pandemic of social media panic travels faster than the COVID-19 outbreak. Journal of Travel Medicine, 27(3), taaa031. https://doi.org/10.1093/jtm/taaa031/5775501

Mesquita, K., Ruão, T., y Andrade, J. G. (2020). Covid-19 pandemic, communication and relationship: an analysis of the social media of the University of Minho. Revista Comunicando, 9, 31-56.

Ministerio Salud da Argentina (2021) Home. (Perfil de Instagram). Instagram. https://www.instagram.com/msalnacion

Ministerio Salud y Deportes Bolivia (2021) Home. (Perfil de Instagram). Instagram. https://www.instagram.com/saluddeportesbo/

Ministerio Salud Brasil (2021) Home. (Perfil de Instagram). Instagram. https://www.instagram.com/minsaude/

Ministerio Salud Colombia (2021) Home. (Perfil de Instagram). Instagram. .https://www.instagram.com/minsaludcol/

Ministerio Salud Chile (2021) Home. (Perfil de Instagram). Instagram. https://www.instagram.com/ministeriosalud/

Ministerio Salud Perú (2021) Home. (Perfil de Instagram). Instagram. https://www.instagram.com/minsa_peru/

Ministerio Salud Paraguay (2021) Home. (Perfil de Instagram). Instagram. https://www.instagram.com/msaludpy/

Ministerio Salud Uruguay (2021) Home. (Perfil de Instagram). Instagram. https://www.instagram.com/mspuruguay/ 
Ministerio Salud Venezuela (2021) Home. (Perfil de Instagram). Instagram. https://www.instagram.com/mppsalud_vzla/

Miola, E., y Marques, F. P. J. (2020). Comunicação pública do Ministério da Saúde no Facebook: uma análise das campanhas contra o Aedes aegypti no "verão do Zika." Revista Eletrônica de Comunicação, Informação e Inovação Em Saúde, 14(1), 34-50. https://doi.org/10.29397/reciis.v14i1.1802

Moorhead, S. A., Hazlett, D. E., Harrison, L., Carroll, J. K., Irwin, A., y Hoving, C. (2013). A new dimension of health care: Systematic review of the uses, benefits, and limitations of social media for health communication. Journal of Medical Internet Research, 15(4), 1-17. https://doi.org/10.2196/jmir.1933

Muralidhara, S., y Paul, M. J. (2018). \#Healthy Selfies: Exploration of Health Topics on Instagram. JMIR Public Health and Surveillance, 4(2), e10150. https://doi.org/10.2196/10150

Njlitics. (2021, 10 de marzo). Perfil de los ministerios de la salud. Njlitics. https://business.notjustanalytics.com/plus/minsaude

Novillo-Ortiz, D., y Hernández-Pérez, T. (2017). Social media in public health: An analysis of national health authorities and leading causes of death in Spanish-speaking Latin American and Caribbean countries. BMC Medical Informatics and Decision Making, 17(1), 1-12. https://doi.org/10.1186/s12911-017-0411-y

O’Donnell, N. H., y Willoughby, J. F. (2017). Photo-sharing social media for eHealth: analysing perceived message effectiveness of sexual health information on Instagram. Journal of Visual Communication in Medicine, 40(4), 149-159. https://doi.org/10.1080/17453054.2017.1384995

Organización Panamericana de Salud (2020a). Plano Estratégico da Organização Pan-Americana da Saúde 2020-2025: Equidade, o coração da saúde. https://iris.paho.org/handle/10665.2/52968

Organización Panamericana de Salud (2020b). COVID-19: Chloroquine and hydroxychloroquine research. Rapid Review - March 28th, 2020, 1-28. https://iris.paho.org/bitstream/handle/10665.2/52094/PAHOEIHKTCOVID19200001_eng.pdf?sequence=1yisAllowed=y

Pinto, P. A., Antunes, M. J. L., y Almeida, A. M. P. (2020, 24 - 27 de junio) Instagram as a communication tool in public health: A systematic review (ponencia) 15a Iberian Conference on Information Systems and Technologies. Universidad de Castilla-La Mancha. Sevilla, España. https://doi.org/10.23919/CISTI49556.2020.9140809

Pinto, P. A., Brasileiro, F. S., Antunes, M. J., y Almeida, A. M. P. (2020). COVID-19 no Instagram: práticas de comunicação estratégica das autoridades de saúde durante a pandemia. Comunicação Pública, 15(29), 1-18. https://doi.org/https://doi.. org/10.4000/cp.11288

Pinto, P. A., Antunes, M. J.L., y Almeida, A. M.P. (2021). Public Health on Instagram: an analysis of health promotion strategies of Portugal and Brazil. Procedia Computer Science, 181, 231-238. https://doi.org/10.1016/j.procs.2021.01.142

Ramanadhan, S., Mendez, S. R., Rao, M., y Viswanath, K. (2013). Social media use by community-based organizations conducting health promotion: a content analysis. BMC Public Health, 13(1129), 110. https://doi:10.1186/1471-2458-13-1129

Rudd, R., y Baur, C. (2020). Health literacy and early insights during a pandemic. Journal of Communication in Healthcare, 13(1), 13-16. https://doi.org/10.1080/17538068.2020.1760622

Santarossa, S., y Woodruff, S. J. (2018). \#LancerHealth: Using Twitter and Instagram as a tool in a campus wide health promotion initiative. Journal of Public Health Research, 7(1), 9-13. https://doi.org/10.4081/jphr.2018.1166

Seltzer, E. K., Jean, N. S., Kramer-Golinkoff, E., Asch, D. A., y Merchant, R. M. (2015). The content of social media's shared images about Ebola: A retrospective study. Public Health, 129(9), 12731277. https://doi.org/10.1016/j.puhe.2015.07.025 
Sendra-Toset, A., y Farré-Coma, J. (2016). Las instituciones de salud pública y las redes sociales: una revisión sistemática. Revista Española De Comunicación En Salud, 7(2), 285-299. https://doi.org/10.20318/recs.2016.3452

Serafinelli, E. (2018). Digital Life on Instagram: New Social Communication of Photography. Emerald Publishing Limited.

Silva, L. B. S., Aragão, C. A., y Pretto, N. D. L. (2021). MacBride report: re-reading in light of threats to the right to communication on digital platforms. Ámbitos. Revista Internacional de Comunicación, 51, 98-115. https://doi: 10.12795/ Ambitos.2021.i51.07

Soares, F. B., Recuero, R., Viegas, P., Bonoto, C., y Hüttner, L. R. (2021). Cloroquina e desinformação sobre Covid-19 na mídia social do Brasil. En C. Martins y A. T. Freitas (Eds.), Pesquisas comunicacionais em interface com arte, tecnologia, religião, meio ambiente (pp.273-292). Pimenta Cultural. https://doi.org/DOI: 10.31560/pimentacultural/2021.854.273-292

We Are Social - Hootsuite. (2021, 5 de marzo). The Digital 2021 Global Overview Report. We Are Social. https://wearesocial-net.s3.amazonaws.com/wp-content/uploads/2021/01/10-Reasons-for-Usingthe-Internet-DataReportal-20210126-Digital-2021-Global-Overview-Report-Slide-48.png

Statista. (2019). Number of monthly active Instagram users 2013-2018. Statista. https://www.statista.com/statistics/253577/number-of-monthly-active-instagram-users/

Statista. (2020). Instagram Age $2020 . \quad$ Statista. https://www.statista.com/statistics/325587/instagram-global-age-group/

Statista. (2021a). Distribution of Instagram users worldwide as of January 2021, by age group. Statista. https://www.statista.com/statistics/325587/instagram-global-age-group/

Statista. (2021b). Percentage of population actively using social media in Latin America. Statista. https://www.statista.com/statistics/454805/latam-social-media-reach-country/

Tang, L., Bie, B., Park, S. E., y Zhi, D. (2018). Social media and outbreaks of emerging infectious diseases: A systematic review of literature. American Journal of Infection Control, 46(9), 962-972. https://doi.org/10.1016/j.ajic.2018.02.010

Teoh, D., Shaikh, R., Schnaith, A., Lou, E., McRee, A. L., Nagler, R. H., y Vogel, R. I. (2019). Evaluation of graphic messages to promote human papillomavirus vaccination among young adults: A statewide cross-sectional survey. Preventive Medicine Reports, 13, 256-261. https://doi.org/10.1016/j.pmedr.2019.01.002

Tursunbayeva, A., Franco, M., y Pagliari, C. (2017). Use of social media for e-Government in the public health sector: A systematic review of published studies. Government Information Quarterly, 34(2), 270-282. https://doi.org/10.1016/j.giq.2017.04.001

van Dijck, J., Nieborg, D., y Poell, T. (2019). Reframing platform power. Internet Policy Review, 8(2), 118. https://doi.org/10.14763/2019.2.1414

Vassallo, A. J., Kelly, B., Zhang, L., Wang, Z., Young, S., y Freeman, B. (2018). Junk Food Marketing on Instagram: Content Analysis. JMIR Public Health and Surveillance, 4(2), e54. https://doi.org/10.2196/publichealth.9594

Villegas-Tripiana, I., Villalba-Diaz, A., y López-Villegas, A. (2020). Análisis de la información sobre COVID19 en sitios web de organizaciones públicas sanitarias. Revista Española De Comunicación En Salud, 1, 234. https://doi.org/10.20318/recs.2020.5437

Viswanath, K., Lee, E. W. J., y Pinnamaneni, R. (2020). We Need the Lens of Equity in COVID-19 Communication. Health Communication, 35(14), 1743-1746. https://doi.org/10.1080/10410236.2020.1837445

World Health Organization (2018). Comunicação de riscos em emergências de saúde pública: Um guia da WHO para políticas e práticas em comunicação de risco de emergência. World Health Organization. 


\section{Semblanza de los autores}

Pâmela Araujo Pinto realiza su investigación posdoctoral en el Departamento de Comunicación y Arte (Universidad de Aveiro). Se graduó en Periodismo por la Universidad Federal de Maranhão (2007) y obtuvo su doctorado en Comunicación en la Universidad Federal Fluminense (2015). Es colaboradora del Centro de Investigación DigiMedia. Sus intereses de investigación están relacionados con la comunicación y la salud pública, la comunicación institucional y los medios sociales, con énfasis en Instagram, y los medios regionales.

Maria João Antunes es profesora asistente en el Departamento de Comunicación y Arte de la Universidad de Aveiro (Portugal). Es doctora en Ciencias y Tecnologías de la Comunicación (2007) y licenciada en Nuevas Tecnologías de la Comunicación (1998) por la Universidad de Aveiro. Sus intereses de investigación actuales son los contenidos generados por los usuarios, las tecnologías digitales para mejorar los territorios y el uso de Instagram por parte de las organizaciones.

Ana Margarida Pisco Almeida es doctora en Ciencias y Tecnologías de la Comunicación e imparte clases en el Departamento de Comunicación y Arte de la Universidad de Aveiro, Portugal. Ha desarrollado actividades de investigación relacionadas principalmente con e-salud y bienestar, accesibilidad e inclusión digital, soluciones digitales para necesidades especiales, promoción de la salud a través de las TIC y desarrollo de medios digitales y metodologías de evaluación. Es miembro de la junta ejecutiva del Centro de Investigación DigiMedia, y coordina el grupo 'eHealth and Wellbeing'. 